\title{
A Numerical Study of Three-Dimensional Bénard Convection
}

\author{
Part II. On the Preferred Horizontal Plan Form
}

\author{
By Takeo Kitade \\ Meteorological Research Institute, Tokyo \\ (Manuscript received 27 June 1975, in revised form 16 August 1975)
}

\begin{abstract}
The preferred horizontal plan form of Bénard convection is examined by a numerical integration of Boussinesq system of equations. We carried out the computations of the cases of $R / R c$ $=2$ and 6 for air and water, where $R$ is a prescribed Rayleigh number and $R c$ is the critical Rayleigh number. The steady rolls are obtained under a nearly random small initial temperature perturbation except the case of $R=6 R c$ for water. In the case of $R=6 R c$ for water we have a steady three-dimensional convection. However the final steady convection with a finite amplitude initial perturbation has a different horizontal plan form from one with the nearly random small initial temperature perturbation.

The vertically coherent oscillation whose period is almost the same as that observed in the laboratory experiments by Krishnamurti (1970) and Willis and Deardorff (1970) was found in the case of $R=6 R c$ for air.
\end{abstract}

\section{Introduction}

The state of knowledge of the linear stability theory for Bénard problem is virtually complete (Chandrasekhar, 1961). However there are many problems which we cannot understand by the linear theory. Among those problems the determination of amplitude was treated in Part I of this article and the determination of preferred horizontal plan form is treated in Part II.

An infinite degeneracy of eigensolutions was found in the linear theory for three-dimensional Bénard convection. This implies that the resulting cellular convective flow is not uniquely determined by the linearized equation of motion and the boundary conditions. We should take account of the non-linear terms. Malkus \& Veronis (1958) have shown for special solutions that the degeneracy persists for finite amplitude solutions, too. They showed that flows with rectangular or hexagonal cell pattern are finite amplitude solutions and that the arbitrary ratio of side length of a rectangle is possible. They intended to obtain the preferred cell shape by "relative stability criterion". Schlüter, Lortz and Busse (1965) showed that convecting rolls are the only pattern which is stable to the perturbations just above the critical Rayleigh number " $R c$ ". This result may imply that two-dimensional rolls are preferred horizontal plan form of Bénard problem just above Rc. Newell, Lange and Aucoin (1970) showed that if the initial disturbance field is small and has a sufficiently smooth spectrum, then a natural statistical selection process chooses from the initial disorder a perfectly ordered field of single rolls. However their results may be valid only just above the critical Rayleigh number. In the numerical simulations of Bénard convection, Lipps and Somerville (1971), Somerville (1973) and Veltishchev and Zelnin (1975) obtained rolls from a random temperature perturbation in the case of $R \sim 2.5 R_{C}$ for air.

There is some information from experimental studies. Koschmieder (1966) found a system of concentric round rolls with round wall and rectangular patterns, which transformed into rolls and combinations of rolls and rectangular cells with a rectangular frame. Krishnamurti (1970, 1973) showed that steady rolls are realized just above the critical Rayleigh number $R c$ and the three-dimensional cellular motions are obtained at higher Rayleigh numbers for several Prandtl numbers from 0.02 to $10^{4}$. However she found that there is hysteresis in the flow pattern as $R$ 
is increased from below or decreased from above, which implies the dependence of cellular pattern in the steady state on the initial conditions.

The theoretical studies have not been able to predict exactly the preferred horizontal plan form of Bénard convection except just above the critical Rayleigh number. The numerical investigations reported so far are limitted to particular combinations of Rayleigh number and Prandtl number. In the present paper we shall examine the preferred horizontal plan form for various values of Rayleigh number and Prandtl number as a initial value problem. In contrast to the earlier numerical studies, in which finite difference method was adopted, we shall treat the problem by a spectral method.

\section{Model}

\section{2-1. The basic equations and boundary conditions}

The basic equations and the method of calculation are the same as those in Part I. The nondimensional Boussinesq equations are written as follows,

$$
\begin{aligned}
& \left(\frac{\partial}{\partial t}-\sigma \nabla^{2}\right) \boldsymbol{v}=-\boldsymbol{v} \cdot \nabla \boldsymbol{v}-\nabla P+\sigma R T \boldsymbol{k} \\
& \left(\frac{\partial}{\partial t}-\nabla^{2}\right) T=w-\boldsymbol{v} \cdot \nabla T+\frac{\partial \overline{w T}}{\partial z}-w \frac{\partial \bar{T}}{\partial z} \\
& \left(\frac{\partial}{\partial t}-\nabla^{2}\right) \bar{T}=-\frac{\partial \overline{w T}}{\partial z} \\
& \nabla \cdot \boldsymbol{v}=0
\end{aligned}
$$

where $\boldsymbol{v}=(u, v, w)$ is velosity, $p$ is pressure, $T$ is perturbation temperature, $(1+\bar{T}-z)$ is horizontally averaged temperature, $\boldsymbol{k}$ is a unit vector in the $z$-direction, $\sigma$ is Prandtl number, and $R$ is Rayleigh number. The bar represents horizontally averaged value. The boundary conditions are based on the assumptions that the boundaries $(z=0, z=1)$ are perfect conductors of heat and flat and stress-free and lateral boundaries $(x=0$, $X$ and $y=0, Y$ ) are symmetric surface. Then the conditions are

$$
\begin{aligned}
& w=\frac{\partial u}{\partial z}=\frac{\partial v}{\partial z}=\bar{T}=T=0 \text { at } \quad z=0,1 \\
& u=\frac{\partial v}{\partial x}=\frac{\partial w}{\partial x}=\frac{\partial T}{\partial x}=0 \quad \text { at } \quad x=0, X \\
& v=\frac{\partial u}{\partial y}=\frac{\partial w}{\partial y}=\frac{\partial T}{\partial y}=0 \quad \text { at } \quad y=0, Y
\end{aligned}
$$

Therefore a general spatial representation which satisfies the boundary conditions is

$$
\begin{aligned}
& u=\sum U_{k, l, m} \sin \frac{k \pi}{X} x \cos \frac{l \pi}{Y} y \cos m \pi z \\
& v=\sum V_{k, l, m} \cos \frac{k \pi}{X} x \sin \frac{l \pi}{Y} y \cos m \pi z \\
& w=\sum W_{k, l, m} \cos \frac{k \pi}{X} x \cos \frac{l \pi}{Y} y \sin m \pi z \\
& T=\sum^{\prime} T_{k, l, m} \cos \frac{k \pi}{X} x \cos \frac{l \pi}{Y} y \sin m \pi z \\
& p=\sum P_{k, l, m} \cos \frac{k \pi}{X} x \cos \frac{l \pi}{Y} y \cos m \pi z \\
& \bar{T}=\sum^{\prime \prime} \bar{T}_{m} \sin m \pi z
\end{aligned}
$$

where

$$
\begin{gathered}
\sum=\sum_{k=0}^{K} \sum_{l=0}^{L} \sum_{m=0}^{M}, \quad \Sigma^{\prime}=\sum_{k=0}^{K} \sum_{l=0}^{L} \sum_{m=0}^{M}, \quad \Sigma^{\prime \prime}=\sum_{m=0}^{\infty} \\
(k+l \neq 0)
\end{gathered}
$$

If $K, L$ and $M$ are allowed to become infinite, the representation should converge to the rigorous solution because it is expressed by a complete orthogonal set. On expecting fast convergence of the series in our problem, we truncate it with the maximum wavenumber vector $(K, L, M)$. If expression (2-6) is substituted into equations $(2-1),(2-2),(2-3)$ and (2-4) and if we use the orthogonality of trigonometric functions, we derive a set of non-linear ordinary, differential (in time), equations for the amplitudes of the harmonic components. The problem is to find the behavior of the harmonic amplitudes as a function of time for a given value of $\sigma$ and a supercritical value of $R$. These equations are integrated numerically when a suitable set of initial conditions is given.

\section{2-2. Computational domain}

According to the linear theory the preferred horizontal wavenumber at the critical Rayleigh number is given for the boundary condition used in this study by

$$
a_{c}=\sqrt{\left(\frac{k}{X}\right)^{2}+\left(\frac{l}{Y}\right)^{2}}=\sqrt{\frac{1}{2}} .
$$

We select the value of the lateral boundary position $(X, Y)$ so that the mode $(1,1,1)$ is the critical mode, that is, 


$$
\left(\frac{1}{X}\right)^{2}+\left(\frac{1}{Y}\right)^{2}=\frac{1}{2}
$$

where the mode $(k, l, m)$ denotes the component of the perturbation which have wavenumber vector $(k, l, m)$. As the author mentioned in section 1 , the degeneracy of eigensolutions exists in the problem of three-dimensional Bénard convection. It is desired that we select the domain of computation to be able to describe the degeneracy. Therefore we select the size of the domain as follows,

$$
\left(\frac{2}{X}\right)^{2}=\frac{1}{2}
$$

Then the both mode $(2,0,1)$ and mode $(1,1,1)$ have the same critical wavenumber. From (2-7) and $(2-8)$ we have

$$
\left\{\begin{array}{l}
X=2.8284 \\
Y=1.6330
\end{array}\right.
$$

The modes $(2,0,1)$ and $(1,1,1)$ represent the rolls and the rectangular cell, respectively. We have

$$
\begin{aligned}
\cos & \frac{2 \pi}{X} x \cdot \sin \pi z+2 \cos \frac{\pi}{X} x \cdot \cos \frac{\pi}{Y} y \cdot \sin \pi z \\
= & \left\{\cos \frac{2 \pi}{X} x+2 \cos \frac{\pi}{X} x \cdot \cos \frac{\sqrt{3}}{X} \pi y\right\} \\
& \cdot \sin \pi z
\end{aligned}
$$

Therefore if the ratio of amplitude of mode $(1,1,1)$ to that of mode $(2,0,1)$ is 2 , we have a hexiagonal cell (Christopherson, 1940; Chandrasekhar, 1961). Thus we investigate the selection of flow patterns among rolls, rectangular cell and hexiagonal cell, all of which have horizontal wavenumbers of the same magnitude and hence the same stability in the linear theory.

We may expect that the dominant modes in the mature stage are $(2,0,1),(1,1,1),(0,1,1)$, $(2,1,1),(1,0,1)$ which have wavenumbers close to $a_{c}$. In this article the behaviors of five modes above listed will be noticed mainly. The mode which has wavenumber component of sum and difference of those of two modes is generated by non-linear terms and the two modes interact each other through the generated mode. We want to use the model in which such non-linear interaction among modes can be described. Thus we select $K=3, L=2$ and $M=4$. In our truncated model the higher order modes are disregarded. Since the amplitudes of higher order modes, however, decrease abruptly in the model of Bénard convection as shown in Part I, we may expect that the error due to the truncation is not serious.

\section{2-3. The specification of parameters}

Krishnamurti $(1970,1973)$ have found the rolls in the range of $R<R_{I}$ and meta-stable threedimensional convection in the range of $R \geq R_{I}$ in her laboratory experiments. The deviding point $R_{I}$ is about $2.7 R_{C}$ and $4 R_{C}$ in the cases of air and silicon oil, respectively. In order to examine the properties of both convections, we select the value of Rayleigh number as follows,

$$
\left\{\begin{array}{l}
R=2 R_{C} * \\
R=6 R_{C}
\end{array}\right.
$$

Furthermore the experimental results show the dependence of characteristics on Prandtl number. Therefore we adopt the following Prandtl numbers,

$$
\begin{cases}\sigma=0.713 & \text { (for air) } \\ \sigma=6.8 & \text { (for water) }\end{cases}
$$

\section{2-4. Initial condition}

We are interested in the preferred horizontal plan form for various initial conditions. We will consider the two kinds of initial conditions in which the fluid is at rest.

(a) nearly random small temperature perturbation $(r)$

$$
T_{2,0,1}=T_{1,1,1}=T_{0,1,1}=T_{2,1,1}=T_{1,0,1}=0.01^{* *}
$$

The others vanish.

(b) forced finite amplitude temperature perturbation $\left(f_{1,1,1}\right)$

$$
\begin{aligned}
& T_{1,1,1}=0.1, \quad T_{2,0,1}=T_{0,1,1}=T_{2,1,1}=T_{1,0,1} \\
& \quad=0.001
\end{aligned}
$$

The others vanish.

We define $f_{2,0,1}$ in the same way as $f_{1,1,1}$ except $T_{2,0,1}=0.1$ and $T_{1,1,1}=0.001$.

* The critical Rayleigh number is about 1708 in the laboratory experiments in which the upper and lower boundaries are rigid. However $R_{c}$ is 657.5 in our calculation since the upper and lower boundaries are slippery. Therefore the laboratory experiments may be compared with our results at the same value of $R / R_{c}$.

** Temperature is non-dimensionalized by $\Delta T$. Therefore the amplitude of perturbation is a few percents of difference between temperatures in upper and lower boundaries. 


\section{Results of calculation with nearly random initial condition $\boldsymbol{r}$.}

In this section we discuss the preferred horizontal plan form of convection with initial condition $r$. The result of calculations is summerized in Table 1.

Table 1. The horizontal plan form and Nusselt number in the final steady state of our computation with the initial condition $r$.

\begin{tabular}{c|c|c|c}
\hline$R / R_{c}$ & $\begin{array}{c}\text { Prandtl } \\
\text { number }\end{array}$ & $\begin{array}{c}\text { horizontal } \\
\text { plan form }\end{array}$ & $\begin{array}{c}\text { Nusselt } \\
\text { number }\end{array}$ \\
\hline \multirow{2}{*}{2} & 0.713 & roll & 2.16 \\
& 6.8 & roll & 2.16 \\
\hline \multirow{2}{*}{6} & 0.713 & roll & 3.62 \\
& 6.8 & cell & 3.43 \\
\hline
\end{tabular}

\section{3-1. The case of $R=2 R_{C}$}

Fig. 1 shows the time variation of temperature of each mode in the case of $R=2 R_{c}$. In the initial stage the perturbation develops exponentially, as expected by the linear theory. Since the mode $(2,0,1)$ has almost same growth rate as the mode $(1,1,1)$ and the mode $(0,1,1)$, all these perturbations have almost the same amplitude in initial developing stage. The amplitude of a particular mode becomes prominent in mature stage through the effect of non-linear terms. The three-dimensional convection in the initial developing stage changes into two-dimensional rolls in the later stage. Such a transformation from a three-dimensional cell into a two-dimensional roll was observed in experiments by Koschmieder (1966) and Krishnamurti (1968). Krishnamurti (1970) have also found the final steady rolls at rather low Rayleigh number regardless of Prandtl number in her laboratory experiments.

Schlüter, Lortz and Busse (1965) demonstrated mathematically that all three-dimensional convective cells are unstable and only a two-dimensional roll is stable for the perturbation just above $R_{c}$. Newell, Lange and Aucoin (1970) showed that a natural statistical selection process chooses from initial disorder a perfectly ordered field of single rolls if the initial disturbance field is small and has sufficiently smooth spectrum. Lipps and Somerville (1971), Somerville (1973) and Veltishchev and Zelnin (1975) obtained steady rolls from a random temperature perturbation in the case of $R \sim 2.5 R_{c}$ in the numerical simula-

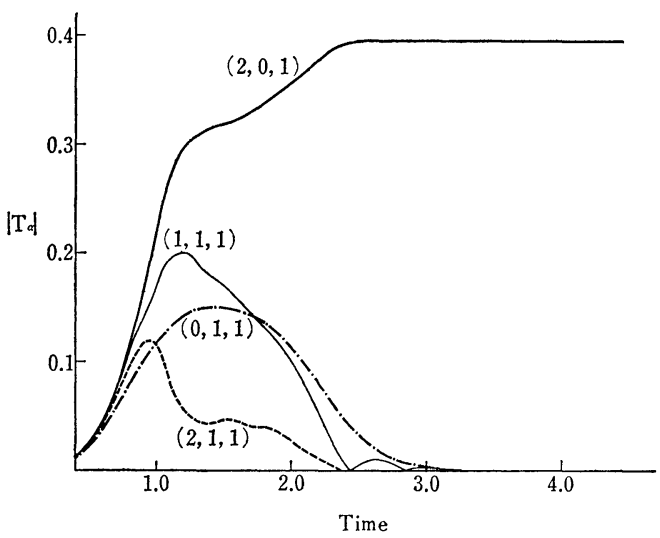

Fig. 1(a). The time variation of absolute value of temperature of each mode in the case of $R=2 R_{c}$ for $\sigma=0.713$ under the initial condition $r$.

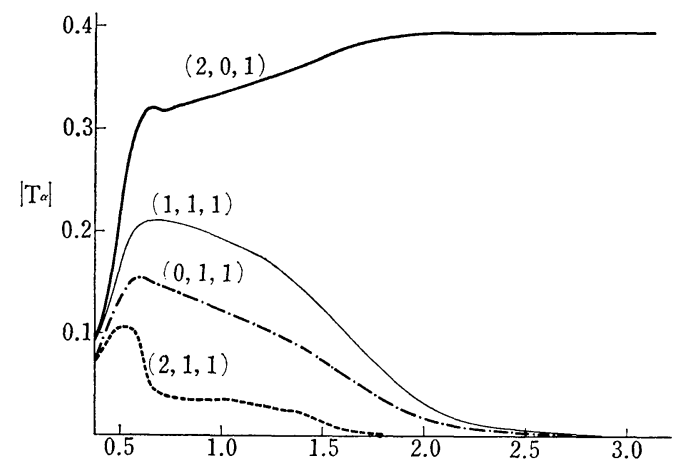

Fig. 1(b). The time variation of absolute value of temperature of each mode in the case of $R=2 R_{c}$ for $\sigma=6.8$ under the initial condition $r$.

tions of three-dimensional Bénard convection for air. Our computation is consistent with their experimental results and theoretical ones.

\section{3-2. The case of $R=6 R_{c}$ and $\sigma=0.713$}

Fig. 2 shows the time variation of temperature of each mode in the case of $R=6 R_{c}$ for air. In the mature stage the mode $(1,1,1)$ oscillates though it damps gradually. The period of oscillation is about 0.36 in a unit $d^{2} / \kappa$, where $d$ is depth of fluid and $\kappa$ is thermal diffusivity. Krishnamurti (1970) and Willis and Deardorff (1970) have found that the period is about 0.27 at $6 R_{c}$ in their laboratory experiments. Photographs and simultaneous temperature measurement by Willis and Deardorff show that the thermal oscillations are associated with lateral oscillations of wavy convection rolls and are 


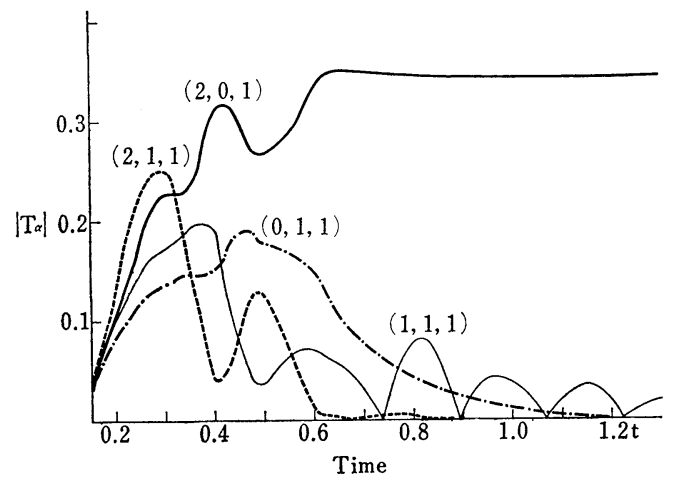

Fig. 2 The same as Fig. 1(a) except for $R=6 R_{c}$.

nearly in phase throughout a vertical line. The numerical experiment for air by Somerville (1973) also reproduced the vertically coherent oscillating convection at $R \sim 5.3 R_{c}$. The oscillation of the mode $(1,1,1)$ in our computation is consistent with the experimental results. Furthermore the experimental results showed that the typical wavelength of wavy roll was $2.5 d$ while that of the roll itself was $3.5 d$. Our numerical model may suppress the oscillation since the given lateral boundary restricts the freedom of size of convection. This may be a reason of damping of oscillation in our computation. In the later stage of our computation we have steady rolls.

\section{3-3. The case of $R=6 R_{c}$ and $\sigma=6.8$.}

Fig. 3 shows the time variation of temperature of main modes in the case of $R=6 R_{c}$ for water. We find no oscillation in mature stage for water unlike the case for air. We have steady threedimensional convection. Fig. 4 shows the horizontal patterns of vertical motion and temperature at $z=0.5$. This pattern is similar to the one in the numerical simulation of Bénard convection for water at $R \sim 4.9 R_{c}$ by Lipps and Somerville

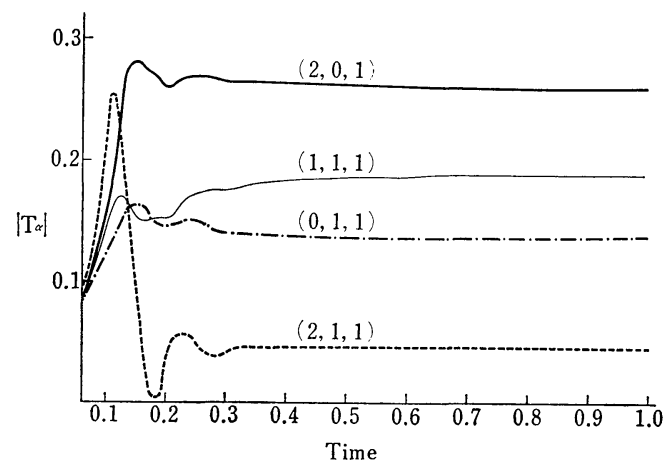

Fig. 3 The same as Fig. 1(b) except for $R=6 R_{c}$.

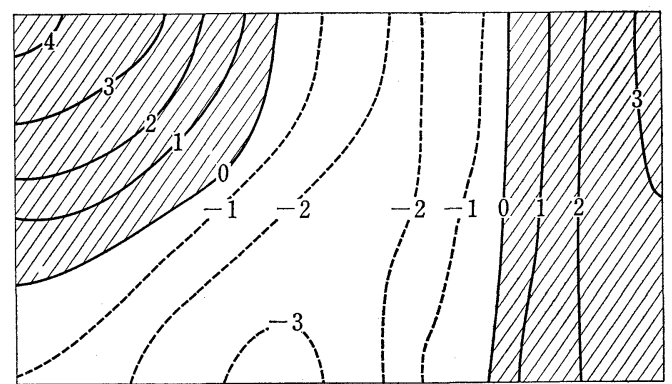

Fig. 4(a) The horizontal pattern of vertical motion of the final steady convection at $z=0.5$ in the case of $R=6 R_{c}$ and $\sigma=6.8$. Values are in units of 10 . The shaded area denotes the area with positive value.

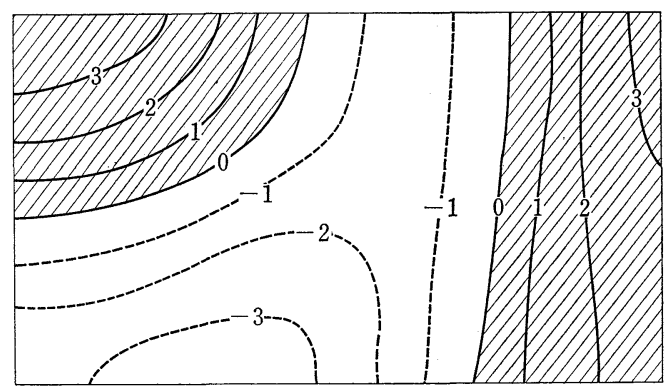

Fig. 4(b) The same as Fig. 4(a) except for horizontal pattern of temperature in units of $10^{-1}$.

(1971). Krishnamurti (1970) has found the steady rolls for water at $R=6 R_{c}$ in her experiments in which Rayleigh number increases very slowly from below. However she suggested that the three-dimensional convection appears when Rayleigh number decreases from above. Since three-dimensional convection appears in the initial developing stage in our numerical experiments, our results may correspond to Krishnamurti's latter result.

\section{The results of calculation with forced initial condition $\boldsymbol{f}_{k, l, m}$}

We will discuss the hysteresis effect on the preferred horizontal plan form, which is found in many laboratory experiments of Bénard convection. The result of calculations is summerized in Table 2.

\section{4-1. The case of $R=2 R_{c}$}

We have a steady roll under the initial condition $r$ in the case of $R=2 R_{c}$. In order to examine the hysteresis of horizontal plan form, we em- 
Table 2. The horizontal plan form and Nusselt number in the final state of our computation with the initial condition $\mathrm{f}_{k, l, m}$.

\begin{tabular}{|c|c|c|c|c|}
\hline$R / R_{c}$ & $\begin{array}{l}\text { Prandtl } \\
\text { number }\end{array}$ & $\begin{array}{c}\text { initial } \\
\text { condition }\end{array}$ & $\begin{array}{l}\text { horizontal } \\
\text { plan form }\end{array}$ & $\begin{array}{l}\text { Nusselt } \\
\text { number }\end{array}$ \\
\hline \multirow{2}{*}{2} & 0.713 & $f_{1,1,1}$ & cell & 1. 96 \\
\hline & 6.8 & $f_{1,1,1}$ & cell & 2.06 \\
\hline \multirow{2}{*}{6} & 0.713 & $f_{1,1,1}$ & cell & 3. 23 \\
\hline & 6.8 & $f_{2,0,1}$ & roll & 3. 63 \\
\hline
\end{tabular}

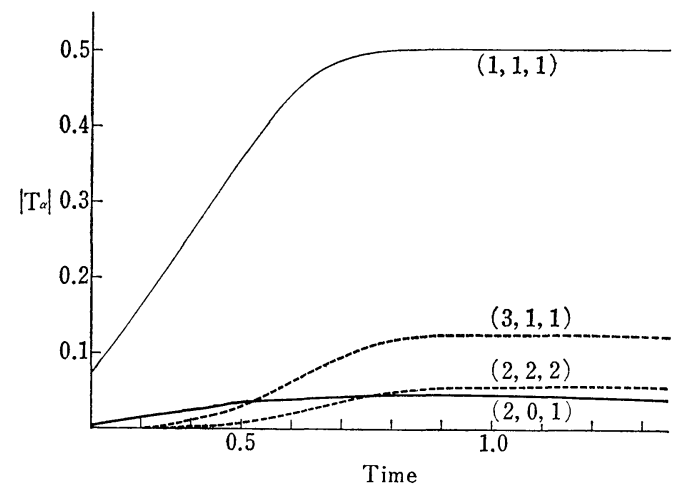

Fig. 5(a) The same as Fig. 1(a) except for the initial condition $f_{1,1,1}$.

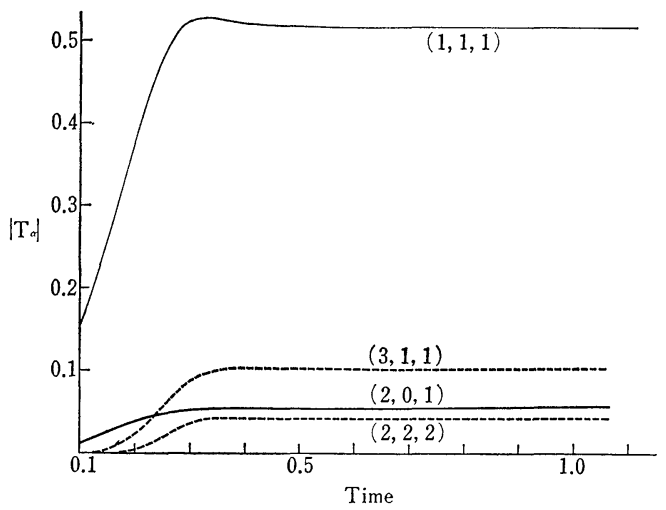

Fig. 5(b) The same as Fig. 1(b) except for the initial condition $f_{1,1,1}$.

ploy the initial condition $f_{1,1,1}$ which gives the finite amplitude three-dimensional convection. Fig. 5 shows the time variation of temperature of each mode. The large amplitude of the mode $(1,1,1)$ follows the change of horizontally averaged temperature and other main modes are suppressed. We have the steady three-dimensional convection in the final stage, too. The present result implys that the steady three-dimensional solution is stable to small perturbations represented by the modes $(2,0,1),(0,1,1),(1,0,1),(2,1,1)$ and others.

Schlüter, Lortz and Busse stated that all the steady three-dimensional solutions are unstable to perturbations. Our result is contradictory to theirs. This may be due to the difference of Rayleigh numbers between the two studies. The discussion by Schlüter, Lortz and Busse is valid only at Rayleigh number close to $R_{c}$. According to the experimental work the hysteresis exists in the planform of the convection at moderate Rayleigh number. Therefore we must treat it as an instability problem of stationary convective motion to finite amplitude perturbations. In the finite amplitude instability problem, the result should depend upon the amplitudes of the perturbations. The experimental work by Krishnamurti suggests unlike our result that the threedimensional convection is unstable to the twodimensional perturbation in the range of $R<4 R_{c}$ for water. The discrepancy between the experimental result and ours may be due to the difference of amplitudes of noise disturbances between the experimental and numerical works.

\section{4-2. The case of $R=6 R_{c}$ and $\sigma=0.713$}

In this case we have a steady roll under the initial condition $r$, so that the initial condition $f_{1,1,1}$ was employed. Fig. 6 shows the time variation of temperature of each mode. In the final stage we have a stationary three-dimensional convection. The existence of hysteresis is clear in this case though it is not reported in laboratory experiments for air.

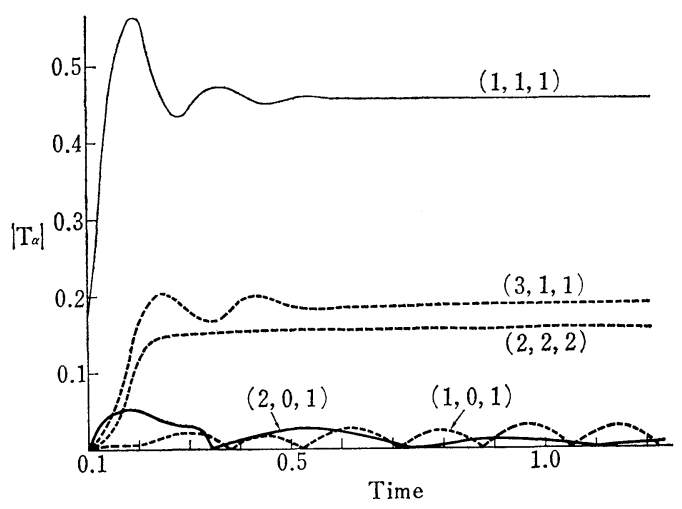

Fig. 6 The same as Fig. 1(a) except for the case of $R=6 R_{c}$ under the initial condition $f_{1,1,1}$.

It should be noticed that the stationary oscillation appears in this case. The period of the oscillation is the almost same as the case with initial condition $r$. The oscillation of the mode $(1,0,1)$ implys that it is in phase throughout a 
vertical line, which is consistent with the experimental result by Willis and Deardorff. However the amplitude of oscillations seems to be much smaller than that observed in the laboratory experiments.

\section{4-3. The case of $R=6 R_{c}$ and $\sigma=6.8$}

We had a steady three-dimensional convection with initial condition $r$. Therefore we employs the initial condition $f_{2,0,1}$ in this case. The steady two-dimensional roll is obtained as final state as shown in Fig. 7. The hysteresis is also found for water, which is found in the laboratory experiment by Krishnamurti.

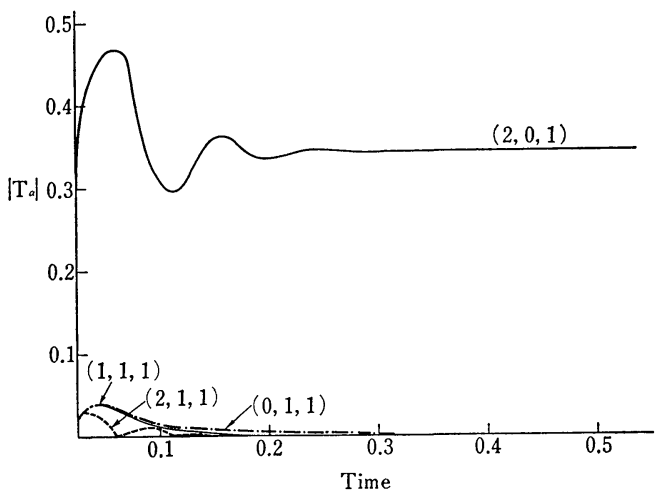

Fig. 7 The same as Fig. 1(a) except for the case of $R=6 R_{c}$ under the initial condition $f_{2,0,1}$.

The non-existence of oscillation in this case is also consistent with the experimental work.

\section{Concluding remarks}

A numerical integration of the Boussinesq system of equations was carried out in order to examine the preferred horizontal plan form of Bénard convection. We have found the preferrence of roll at a rather small Rayleigh number under the initial small perturbation, the hysteresis phenomena for finite amplitude initial conditions and occurrence of oscillation for air.

We calculated the Nusselt numbers in Part I for the roll and the rectangular cells, which have the same wavelength as the ones treated in the present article. According to the result the Nusselt number for roll is larger than that for rectangular cell except the case of $R=6 R_{c}$ for water. Our present result of the cases with small initial perturbation seems to satisfy "the maximum heat flux assumption" by Malkus (1954). However the three-dimensional convection obtained under the small perturbation in the case of $R=6 R_{c}$ for water has smaller Nusselt number than that of roll obtained under the forced finite amplitude initial condition (see Table 1 and 2). Therefore our result does not always satisfy "the maximum heatflux assumption". This result may be connected with the hysteresis effect of finite amplitude convection.

The result of our computations gives a information for the preferred roll size, that is, the present model includes the rolls whose wavelengthes are 5.657, 3.266, 2.828, 1.885 and 1.633. Our result shows that the roll with the wavelength $=2.828$ is selected among those rolls. This problem will be examined in detail by the calculations of convection in the different horizontal domain in Part III of this article.

In this study we found some oscillations in the case of $R=6 R_{c}$ for air. The oscillation in the experimental works is similar to ours in the period and the vertical phase change. However in our model the oscillation damps or the amplitude is much smaller than the experimental counterpart. The reason may be that the oscillation is suppressed by the given lateral boundary in our study. We will examine the oscillation of convection in the different computational domain in the forthcoming paper.

\section{Acknowledgements}

The author wishes to express his hearty gratitude to Prof. T. Matsuno, Tokyo University, who read the manuscript carefully and gave him valuable comments to improve the paper.

\section{References}

Chandrasekhar, S., 1961: Hydrodynamic and hydromagnetic stability, Oxford University Press.

Christopherson, D.G., 1940: Note on the vibration of membrances. Quart. J. Math., 11, 63-65.

Kitade, T., 1974: A numerical study of three-dimensional convection, Part. I, The evaluation of the amplitude. J. Meteor. Soc. Japan, 52, 463-480.

Koschmieder, E.L., 1966: On convection on a uniformly heated plane. Beitr. phy. Atmos., 39, 1-11.

Krishnamurti, R., 1968: Finite amplitude convection with changing mean temperature. Part. 1, Theory., Part. 2, An experimental test of the theory. $J$. Fluid Mech., 33, 445-455, 457-463.

1970: On the transition to turbulent convection. Part. 1, The transition from two-tothree-dimensional flow. Part. 2, The transition to time-dependent flow. J. Fluid Mech., 42, 295-307, 
309-320.

1973: Some further studies on the transition to turbulent convection. J. Fluid Mech., 60, 285-303.

Malkus, W.V.R., 1954: The heat transport and spectrum of thermal turbulence. Proc. Roy. Soc. A., 225, 196-212.

plitude cellular convection. J. Fluid Mech., 4 225-260.

Lipps, F.B., and R.C.J. Somerville, 1971: Dynamics of variable wavelength in finite-amplitude Bénard convection. Phy. Fluids, 14, 759-765.

Newell. A.C., C.G. Lange, and P.J. Aucoin, 1970: Random convection. J. Fluid Mech., 40, 513-
542.

Schlüter, A., D. Lortz, and F. Busse, 1965: On the stability of steady finite amplitude convection. $J$. Fluid Mech., 23, 129-144.

Somerville, R.C.J., 1973: Numerical simulation of small scale thermal convection in the atmosphere. Proc. Third Intern. Conf. Numerical Methods in Fluid Dynamics, Vol. II, Springer Verlag (Lecture Notes in Physics, Vol. 19, 238-245).

Veltishchev, N.F., and A.A. Zelnin, 1975: Numerical simulation of cellular convection in air. J. Fluid Mech., 68, 353-368.

Willis, G.E., and J.W. Deardorff, 1970: The oscillatory motions of Rayleigh convection. J. Fluid Mech., 44, 661-672.

\title{
3 次元ベナード対流の数值的な研究
}

（II）卓越しやすい対流セルの形について

\author{
北出 武夫 \\ 気象研究所
}

ベナール対流の卓越しやすいセルの形を 3 次元 Boussinesq 方程式系の数值時間積分によって調べた. Rayleigh 数 $R$ がその臨界值 $R_{c}$ の 2 倍と 6 倍の場合に空気と水に対して調べた. 小さくてランダムな初期温度擾乱を与充た場合 には, $R=6 R_{c}$ で水に対する場合以外にロール状の定常な対流が得られた。 $R=6 R_{c}$ で水に対する場合には, 定常 な 3 次元のセル状対流が得られた。しかし初期に有限振幅を持つ擾乱を与えた場合には，小さな初期擾乱を与光た場 合とは異なるセルの形が得られた。

$R=6 R_{c}$ で空気に対しては，その周期と垂直方向の位相に拈いて Krishnamurti (1970) や Willis and Deardorff （1970）等による室内実験で得られるものと似た振動する対流が見い出された. 